\title{
Mapping of human arm impedance characteristics in spatial movements
}

\author{
Tasnuva Tabashhum Choudhury ${ }^{1}$, Md Mozasser Rahman², Md Raisuddin Khan ${ }^{3}$, Fazlur Rashid ${ }^{1}$, Md. \\ Rabiul Islam Sarker ${ }^{1}$ \\ ${ }^{1}$ Department of Mechanical Engineering, Faculty of Mechanical Engineering, Rajshahi University of Engineering and Technology, \\ Dhaka Hwy, Rajshahi, Bangladesh \\ ${ }^{2}$ Department of Mechanical Engineering Technology, Faculty of Engineering Technology, Universiti Tun Hussein Onn Malaysia, Johor, \\ Malaysia \\ ${ }^{3}$ Department of Mechatronics Engineering, Kulliyyah of Engineering, International Islamic University Malaysia, Selangor, Malaysia
}

\section{Article Info \\ Article history: \\ Received Aug 31, 2021 \\ Revised Oct 28, 2021 \\ Accepted Dec 16, 2021}

\section{Keywords:}

Human arm

Impedance

Mass-spring-damper

Spatial movement

Trajectory

\begin{abstract}
In this paper, impedance characteristics are determined for discrete movement. It explores the changes in impedance characteristics of human arm during a complete task. This study considered 3D spatial movement for horizontal adduction and abduction. The human arm is considered as massspring-damper system and the modelling is done accordingly. The model is solved for 3 degree of freedom (3DoF) spatial movement usually used for daily work. Inertia, stiffness and damping factor are the impedance characteristics considered in the model. Using position measuring device, the position data of elbow and wrist were obtained with respect to predefined references. The data were used to calculate velocity, acceleration and force. Then, the impedance characteristics were determined by solving the equation of motion of the mass-spring-damper system corresponding to different position of the wrist on the trajectory. These impedance factors were then plotted to map the characteristics. The mappings were done for both total durations to complete task and for segments of the task. The impedance characteristics were observed to vary according to the movement of the arm. Significant changes of damping factor and stiffness were found to occur at the beginning and end of the arm movement.
\end{abstract}

This is an open access article under the CC BY-SA license.

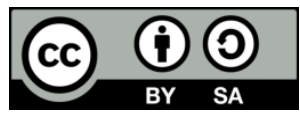

\section{Corresponding Author:}

Md Mozasser Rahman

Department of Mechanical Engineering Technology, Faculty of Engineering Technology

Universiti Tun Hussein Onn Malaysia, Pagoh Campus

Km 1, Jalan Panchor, 84600 Pagoh, Muar, Johor Darul Ta'zim, Malaysia

Email: mozasser@uthm.edu.my

\section{INTRODUCTION}

Human arm movement is an emerging field which seeks to study how human arm works. In such research, dynamic impedance characteristics are mapped against time to have a picture of the dynamics of arm movement in space. The impedance characteristics are then used for designing robotic arm exoskeleton for rehabilitation. However, only few works have shown the dynamic changes of impedance characteristics for spatial movements. Humans have excellent abilities to manipulate objects as various task dynamics are adapted by the central nervous system. For example, for the first time due to some friction, one may have difficulty in opening a door. However, after one or two trials, the appropriate force is applied, and without difficulty one will open the door even without thinking about it. With the help of tools, many tasks performed by humans are essentially unstable [1]. Consequently, there is the need for continuous acquisition of additional skills since in unstable tasks dynamics, different initial conditions, neuromotor noise [2], or any 
type of external perturbation can lead to unsuccessful and inconsistencies in the performance. Understanding how humans interact with the environment can provide insight into the neural mechanisms of adaptation and motor learning with potential application to computer animation of human motion. This will aid the development of robots collaborating with human operators and neuromotor rehabilitations. Conversely, humanlike control strategies are better employed in the improvement of robotic control especially with respect to safety [3].

Significant advances in medical robotics have been made recently (Healthcare robots 2021), and in micro and nanotechnology and teleportation [4], all of which rely on haptic interfaces to allow the human operator manipulate objects not directly accessible to the hand. The safety and performance of such systems significantly depend on motion stability i.e., the coupled stability of the arm interacting with the environment [5]. To properly control these types of systems, it would be useful to know the mechanical impedance. Mechanical impedance is the resistance to infinitesimal perturbations applied at the hand. The mechanical impedance of the human arm can be calculated from the restoring force to slight perturbations imposed in static positions [6] or during movement [7]-[11]. For a given stable or unstable interaction, it is possible to measure the impedance in the movements [12]. Though, this requires many movements, and in every dynamic interaction, it would be more useful to have a compact model to describe the force and impedance. Impedance is shown to depend on position force [6], [13] and instability [12] but no comprehensive model has been proposed so far. In order to learn more about human motor adaptation, works have investigated the adaptation to stable [14]-[16] and unstable [17]-[19] interactions produced by a haptic interface.

Measurement of human arm impedance characteristics is an emerging field. This is due to numerous issues including the difficulty in the measurement of human kinematic data, the high complexity in the understanding of the working principle of human muscle, many hidden factors responsible for smooth arm movement. For a healthy normal human being, the arm movement needs to be very smooth, precise, and definite. It is obvious that for arm movement, not only the arm muscle is responsible, rather they are governed by the central nervous system (CNS) taking signal from the eyes and other sensory organs. When an arm of a human body is performing a task like picking an object or reaching to a specific point, it uses the five basic senses of the body. It uses the vision mainly to obtain information about the location of the object, and how it can be reached. The vision helps to gather enough information to perform desired tasks. The obtained information then computed by the brain and the whole arm system is given signal accordingly to perform the task. According to this information, the eye sends signals through the CNS to the arm muscles to pick or reach object accordingly. Most research have tried to reveal how the CNS controls the arm impedance and the changes in the impedance during arm movement.

Disabled humans are unable to move their arm according to the CNS instruction due to damaged muscle cells. Due to different reasons, human beings could become partially disabled, and this has informed the development of different types of rehabilitation. This field combines the knowledge of biomedical engineering and mechatronics. Rehabilitation robots rehabilitate and help or assist the disabled people so they could perform any task without the help of any person. These robots are designed, fabricated and carefully attached to human arm so they could work as one with the human body. These robots help disabled persons to perform various functions in daily life. This way, disabled people would not have to rely on others to perform vital tasks.

This paper presents the impedance characteristics of human arm movements and to map the impedance characteristics of human arm in spatial movements. This paper also reveals how impedance characteristics of human arm changes during a complete cycle of specific task. In this research, impedance characteristics of human arm considering spatial movement for horizontal adduction and abduction have been determined experimentally. This work is limited to horizontal adduction and abduction of human arm motion. The motion is 3D and involves fast movement as required for daily works. Simulations were done with different combinations to have better understanding of human arm impedance characteristics. To understand human arm impedance characteristics, it is also necessary to know different fields such as, biomechanics, biomedical engineering, robotics, and human anatomy. Analysis has been done by using MATLAB. These types of robotic systems are utilized in the calculation of human arm travelling trajectory.

\section{RESEARCH METHOD}

In this research, the movements (single and multi-degree of freedom) of human arm in space for various tasks are considered. The upper limb consists of three main parts namely, upper arm, forearm and hand. It is composed of three chain mechanisms, the shoulder girdle, the elbow and the wrist, whose association allow a wide range of combined motion. If the shoulder girdle moves so is the elbow and the hand is moved together accordingly. Here, both active and passive movement is investigated. The active movement refers to the movement when a person moves their arm by themselves while in passive movement, a person cannot move their arm as per their will except with some help. Suitable manipulators (single and 
multi-degree of freedom) are constructed to move the arm and to measure the trajectory of the arm movements. The movements are executed as a compromise of the simultaneous, smooth interpolation of joint angles, discomfort minimization, and straight hand paths. Individual muscle and interaction between them at the time of movement is considered. This is based on various minima models which are applied to the on human arm movements. For example, minimum jerk motion, minimum torque change model, and minimum energy cost model.

The experimental data (position, velocity, acceleration, and force) is analysed by using different variants of Recursive least-square method for mapping the impedance parameter. Finally, experiments are conducted to verify the proposed model. The steps taken in this research to achieve the stated research objectives are summarized thus: The research begins with comprehensive literature review to discover and explore the wide range of biomechanics, mechatronics and human anatomy, and consequently, analyse the related problem in arm impedance characteristics in spatial movement. The data of the elbow and the wrist are taken with the help of sensors. They are then analysed with MATLAB. The data of the active and passive movement are taken mathematically and plotted into graph for better understanding. The obtained plot is used for comparing all the proposed theories. The data are analysed by using different variants of recursive least-squares methods for mapping the impedance parameter.

\subsection{Experimental setup}

The Cartesian positional data in 3D space is measured using an array of fiber optic curvature sensor known as a Shape Tape. This tape is generally available with different numbers of sensors of different sensing zone lengths and with different leads and tip lengths. The schematic of a typical shape tape is as shown in Figure 1. The tape used in this experiment is $1810 \mathrm{~mm}$, leads length is $1080 \mathrm{~mm}$, the length of the sensing zone is $720 \mathrm{~mm}$, and the tip is $10 \mathrm{~mm}$. Its sampling rate is $110 \mathrm{~Hz}$. In Figure 2, the shape tape device is attached to a subject. Data connector is connected to the device by the COM port and to the PC by USB connection. The arm tape used here is Shape Tape plus. For this case, the arm's homing position was the palms facing down. Before acquiring the data, the shape tape reading was verified by moving the device in known environment. Also, it was checked to determine how many data points can be obtained. To be more specific, the length of the sensor portion is $760 \mathrm{~mm}$. It gives the positional data with respect to the settings.

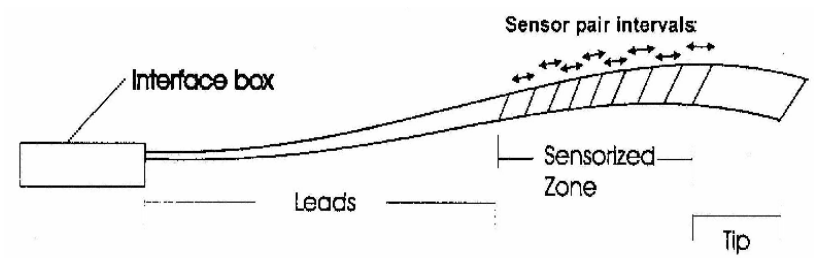

Figure 1. Schematic diagram of shape tape

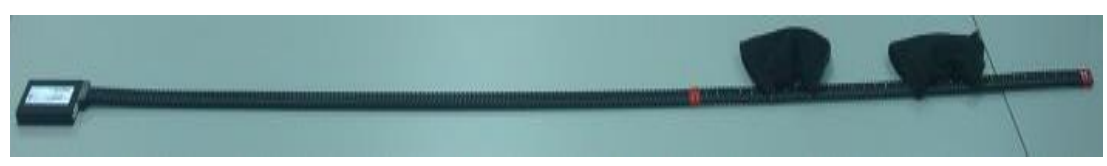

Figure 2. Shape tape device

\subsection{Experimental paradigm}

Six subjects including four females and two males participated in the experiments as shown in Table 1. Participants are ranged between 25-35 years old. None of the subjects had any sensory, perceptual, or any motor disorders. Subjects are not familiar regarding the research topic and had no prior experience of the experiment. Therefore, at first, they are given some instructions and asked to do the trials. In the experiment, both upper and lower arms are considered. The subjects were asked to move their hands in a parallel horizon with the surface of the table for some distance from it. Thus, the subjects were not dragging the mass, rather, they were carrying the weight. For uniformity of the arm trajectory, some indications were plotted on the travelling plane and the subjects were asked to move their arm according to the path. The parameter of subjects is given in the Table 1 . 


\begin{tabular}{ccccc}
\multicolumn{5}{c}{ Table 1. Description of the subjects } \\
\hline Subject & Age & Sex & Length of arm, mm & Length of lower arm, mm \\
\hline Sub 1 & 30 & Female & 60 & 33 \\
Sub 2 & 32 & Female & 54 & 30 \\
Sub 3 & 27 & Male & 59 & 32 \\
Sub 4 & 30 & Female & 61 & 36 \\
Sub 5 & 31 & Female & 56 & 30 \\
Sub 6 & 35 & Male & 56 & 32 \\
\hline
\end{tabular}

\subsection{Data collection}

In this experiment, the subjects were asked to sit on a fixed chair to avoid unwanted body movements as shown in Figure 3. The middle point of the arm was fixed on the table. According to the paradigm, the subject shown in Figure 4 started from point 1 to point 2, and again, returned back to point 1 . This whole procedure is considered as one full cycle. This one cycle was repeated for three times, but not continuously. Due to muscle fatigue and stress, the arm was given some space to take rest. The whole procedure was done periodically for three days. The considered movement is an active daily routine task with some external force applied by handling an object of $1.5 \mathrm{~kg}$ weight.

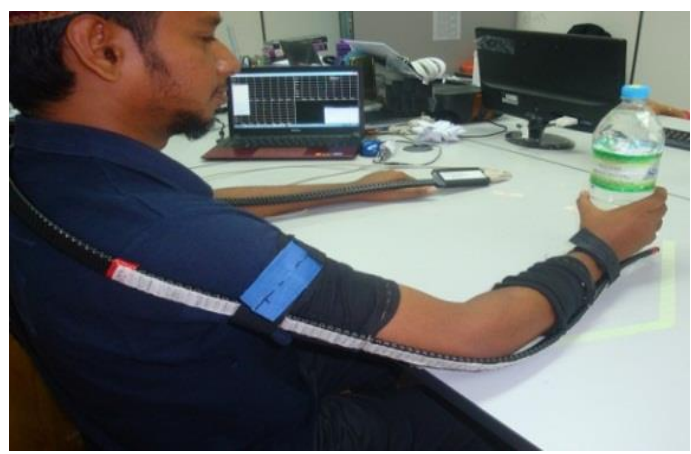

Figure 3. Shape tape mounted on subject's arm

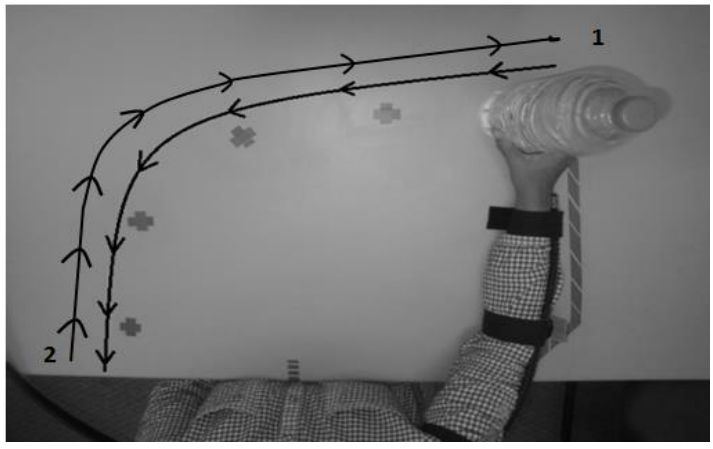

Figure 4. Experiment pathway

\subsection{Data validation}

For the validation of the measured data, at first a kinematic model based on the inverse kinematics for the arm movement on the horizontal surface is developed and compared with the measured data. In Figure 5, the black dotted areas are the coverage of the arm where the arm can swing. The dark line at the right bottom corner of Figure 5 is the measured Cartesian data of the wrist. The arms limited movement is defined by considering the human arm movement limitation. The coloured line indicates the actual positional change of the wrist position. The sample is indicated by the colour bar of the right side which changes colour from blue to red. From the figure, it is shown that the measured data exist in the area where the arm can move. Also, the angular velocity at the shoulder and elbow are also estimated and compared with the theoretical results obtained from inverse kinematics [20]. 
Measured data were used to calculate the angular position of shoulder joint and elbow joint using inverse kinematics [21], [22]. Theoretical angular position of the shoulder and elbow were compared with the calculated data. Data with minimum error were used for further calculation. A sample comparison is shown in Figure 6. The angular change in the shoulder is shown in above and the lower one is for angular change in elbow. In Figure 6, the black line indicates the actual angular position change, the blue line is the angular positional change computed using inverse kinematics and the red line is the error calculated by least square error method.

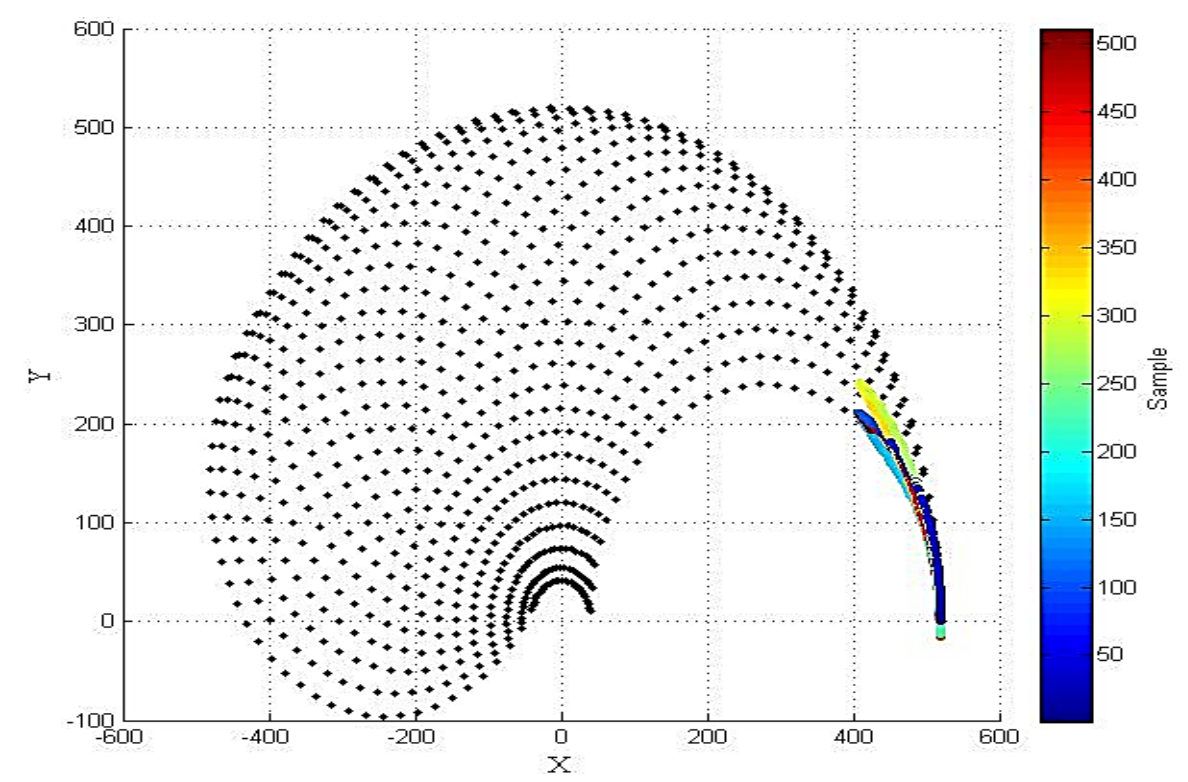

Figure 5. Validation of acquired positional data

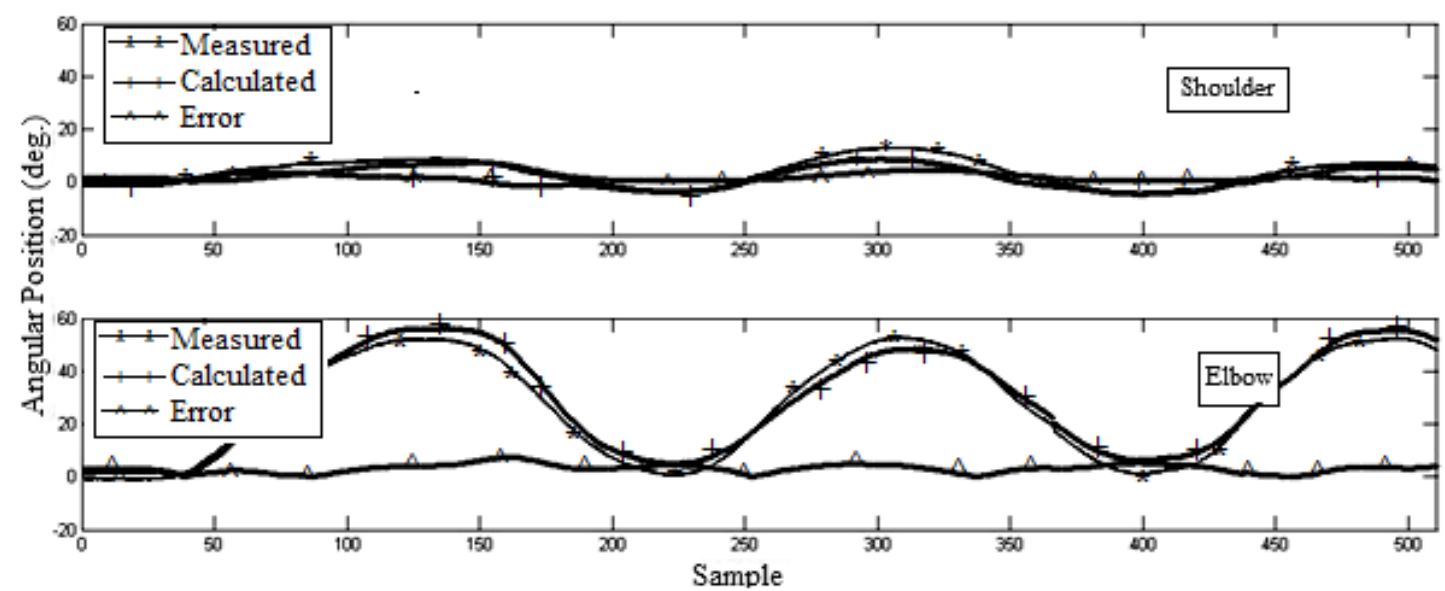

Figure 6. Comparison of angular changes between actual measured angle and the computed angles

\subsection{Mathematical model}

The applied mathematical model in this study is the same as the dynamic equation of motion for mass-spring-damper system. To acquire the kinematic data, Measured Inc., Shape Tape device was used. This device gives the positional value of the desired Cartesian coordinates against time. The developed arm model for the measurement of the dynamic impedance is as shown in Figure 7. The hand impedance model can be expressed for the end-point level when the human arm is under a stable posture by (1).

$$
\mathrm{MX}+\mathrm{BX}+\mathrm{KX}=\mathrm{F}
$$


where $\mathrm{M}, \mathrm{B}, \mathrm{K} \in \mathrm{R}^{(3 \times 3)}$ represent the hand inertia, viscosity and stiffness matrices respectively.

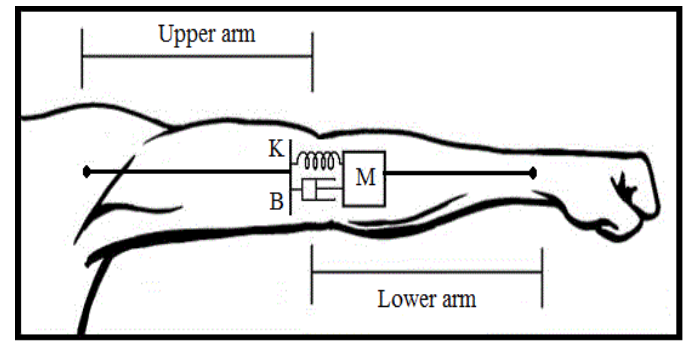

Figure 7. Arm model for dynamic impedance measurement

$\mathrm{X} \in \mathrm{R}^{3}$ and $\mathrm{F} \in \mathrm{R}^{3}$ are the end point position and force vector, respectively. The movement considered in this study is fast movement where some of the terms can be compensated [23]. Here the forces are calculated from the Newton's second law. The position of the hand as well as the end position is measured by Measured Shape Tape ${ }^{\circledR}$ device. Therefore, as shown in (1) is transformed to an identifiable form, with respect to the impedance matrices $\mathrm{M}, \mathrm{B}$ and $\mathrm{K}$ as given in (2).

$$
\mathrm{YI}=\mathrm{F}
$$

where, $I \in R^{27}$ is the parameter vector to be identified, which is given by (3) while the impedance matrix functions are given in (4) to (6).

$$
\begin{aligned}
& I=\left[M_{x}^{T} B_{x}^{T} K_{x}^{T} M_{y}^{T} B_{y}^{T} K_{y}^{T} M_{z}^{T} B_{z}^{T} K_{z}^{T}\right]^{T} \\
& M=\left[M_{x}^{T} M_{y}^{T} M_{z}^{T}\right]^{T} \\
& B=\left[B_{x}^{T} B_{y}^{T} B_{z}^{T}\right]^{T} \\
& K=\left[K_{x}^{T} K_{y}^{T} K_{z}^{T}\right]^{T}
\end{aligned}
$$

$\mathrm{Y}$ in $(2)$ is a $(3 \times 25)$ matrix defined by

$$
\mathrm{Y}=\left[\begin{array}{ccc}
\mathrm{L}^{\mathrm{T}} & 0_{1 \times 9} & 0_{1 \times 9} \\
0_{1 \times 9} & \mathrm{~L}^{\mathrm{T}} & 0_{1 \times 9} \\
0_{1 \times 9} & 0_{1 \times 9} & \mathrm{~L}^{\mathrm{T}}
\end{array}\right]
$$

where, $L \in \mathbb{R}^{9}$ is defined by

$$
\mathrm{L}=\left[\ddot{X}^{T} \dot{X}^{T} \dot{X}^{T}\right]^{\mathrm{T}}
$$

$0_{1 \times 9}$ is a zero matrix of size $(1 \times 9) \dot{X}, \ddot{X}$ vectors representing end-point velocity and acceleration.

According to (2) is used for computing I by using least-square method and $\mathrm{Y}$ is calculated by arm end-point positional data of one complete cycle.

\section{RESULTS AND DISCUSSION}

The main intention of this paper is to investigate the impedance characteristics of the human arm for designing exoskeleton for mimicking the human arm movement. In this study, the movement considered is $3 \mathrm{D}$ and the duration of the movement is about $2 \mathrm{~s}$. As the movement is not discrete point-to-point movement, it is not convenient to represent the impedance characteristics by ellipses. That is why the impedance characteristics against the time (sample) are plotted to see the dynamic changes during the whole process. In the plot, the impedance changes during the complete three cycles are presented. Experiment was conducted using six subjects. Due to the different ergonomic property, they have completed the assigned task 
in different time and covered different workspace. This can be visualized in Figure 8. The data obtained from the experimentations are plotted against both with respect to sample and normalized time to see how the impedances differ from subject to subject and how the impedances change with the arm position with respect to time. A sample of wrist positions of six subjects are plotted against time in Figure 8. From this figure, it is seen that different subjects perform the same task in different time duration. The wrist positions change is plotted in normalized time while it is observed that all the subjects moved their arms following similar trend. It is also seen that subject 6 moved his arm lesser than the others, where subject 2 shows maximum movement.

Figure 9 shows the three cycles of position $X$ for six subjects. Downward curve represent the wrist movement in the forward direction while during the upward curve represent the movement of the wrist in the reverse direction. For all the subjects, the trend of $X$ positions is similar for both forward and reverse motions. It is also seen that the $X$ position maintains a smooth curve all the time which implies there was no jerk during this cyclic motion. The reach of the $X$ position was different for different subjects due to their arm lengths and other argonomics data.

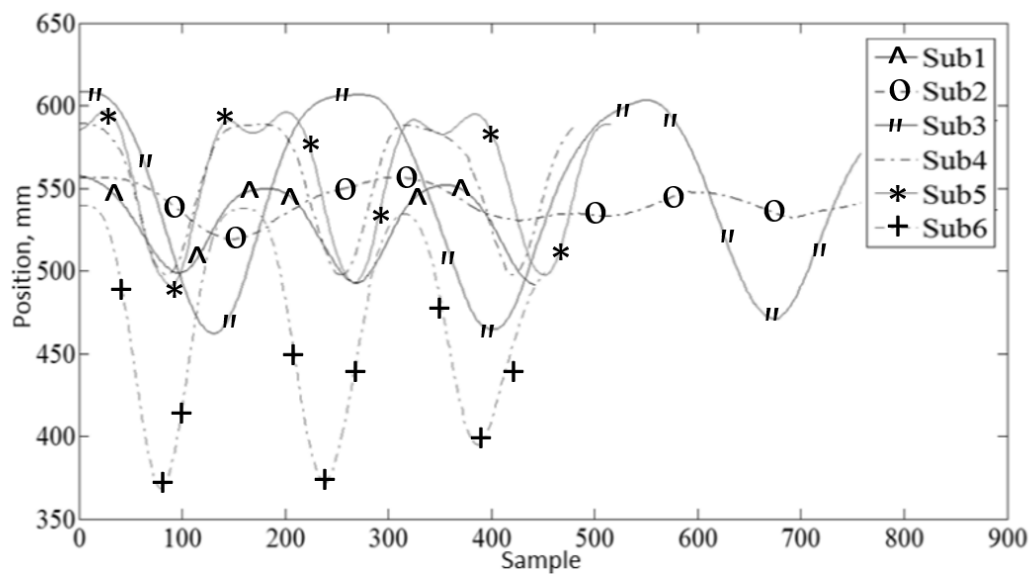

Figure 8. Position change along $\boldsymbol{X}$ direction among the subjects

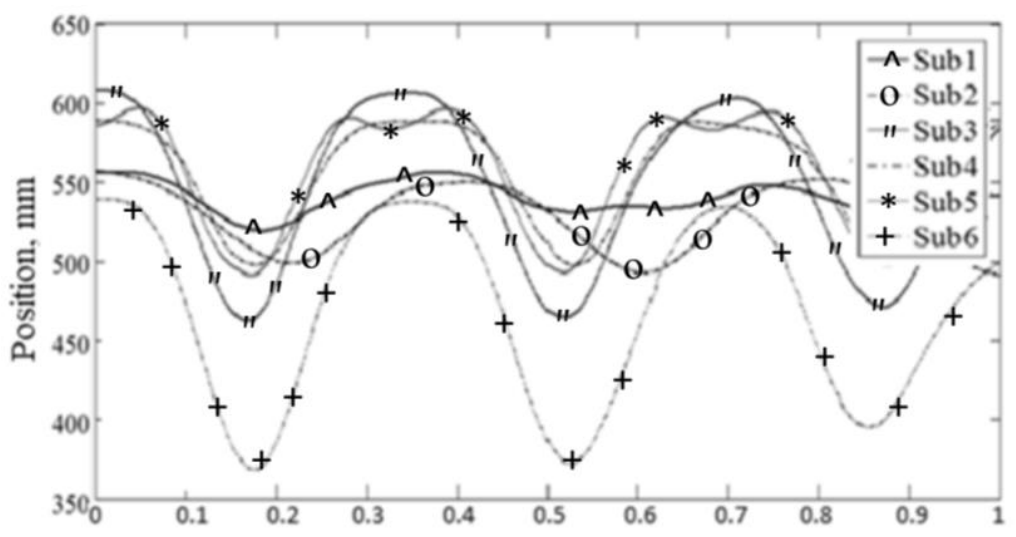

Figure 9. Position plot against normalized time

\subsection{Inertia componenets}

The calculated inertia componemts are shown in Figure 10. From the figure it is seen that inertia change occurs during starting position, turning position and ending position. Moreover, considerable amount of change is seen in the $\mathrm{X}$ and $\mathrm{Y}$ diagonal components of inertia. As the $\mathrm{Z}$ directional movement is very small, the inertia change for all the $\mathrm{Z}$ diagonal components are very small except at starting position. Generally, for a solid object the inertia remains constant during the motion, but in case of muscle with respect to muscle movement muscle shape will be changing. Due to this reason, it changes its center of mass 
and hence in every arm moment their inertia changes. It is shown that the trend is zigzag, and it ranges from $20 \mathrm{~N} . \mathrm{s}^{2} / \mathrm{m}$ to $-20 \mathrm{~N} . \mathrm{s}^{2} / \mathrm{m}$, though around normalized time of 0.9 , it gets out of $40 \mathrm{~N} . \mathrm{s}^{2} / \mathrm{m}$ and $-50 \mathrm{~N} . \mathrm{s}^{2} / \mathrm{m}$. In Figure 10 the considerable amount of change in $X$-diagonal component of inertia is observed at the ending position i.e. starting from position 1 to position 2 and again tending to return to position 1 . From Figures 10 , the maximum inertia is observed by subject 4 and minimum is by subject 1 . The magnitude of the inertia of $X$ diagonal component is larger than the $Y$ and $Z$ diagonal component of inertia.
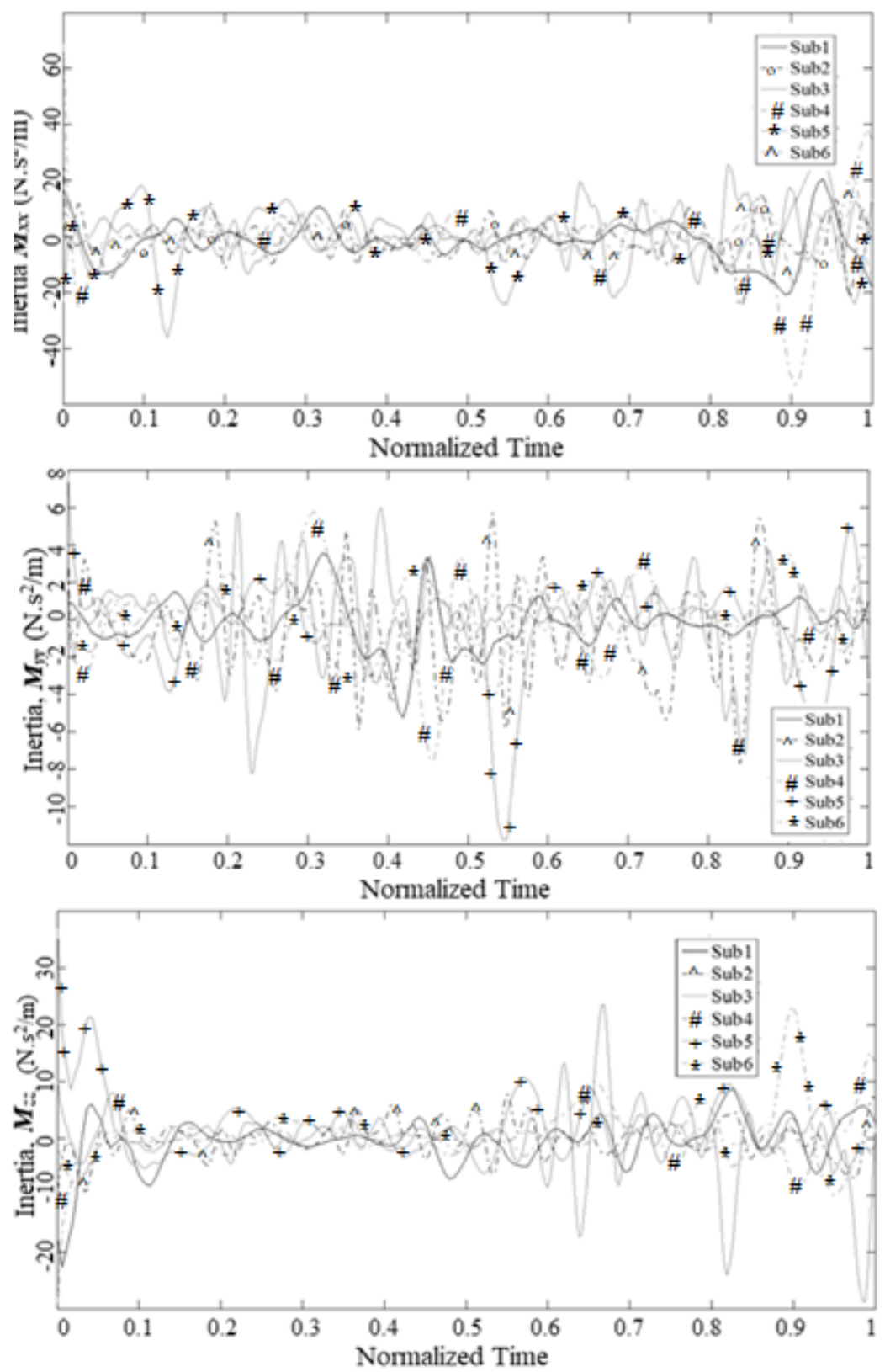

Figure 10. Inertia componemts of all subjects

\subsection{Stiffness componenets}

The stiffness components of all dimension are shown in Figure 11. The maximum stiffness change along $X$ component is observed in position 4 when the subject changes its movement direction and found by comparing with the position graph in Figure 9. The maximum magnitude is $7 \mathrm{~N} / \mathrm{m}$, and the minimum is $-7.5 \mathrm{~N} / \mathrm{m}$ while the maximum deviation is observed by subject 4 (Top graph). The maximum stiffness change is observed by subject 3 while the minimum by subject 1 . The maximum stiffness change along $Y$ component is less than $X$ component. The maximum magnitude is $6 \mathrm{~N} / \mathrm{m}$, and the minimum is $-4.5 \mathrm{~N} / \mathrm{m}$ 
while the maximum deviation is observed by subject 6 . The maximum stiffness change is observed by subject 6 while the minimum also by subject 1 . The stiffness changes along $Z$ component are less than $X$ and $Y$ components as less movements in $Z$ direction.
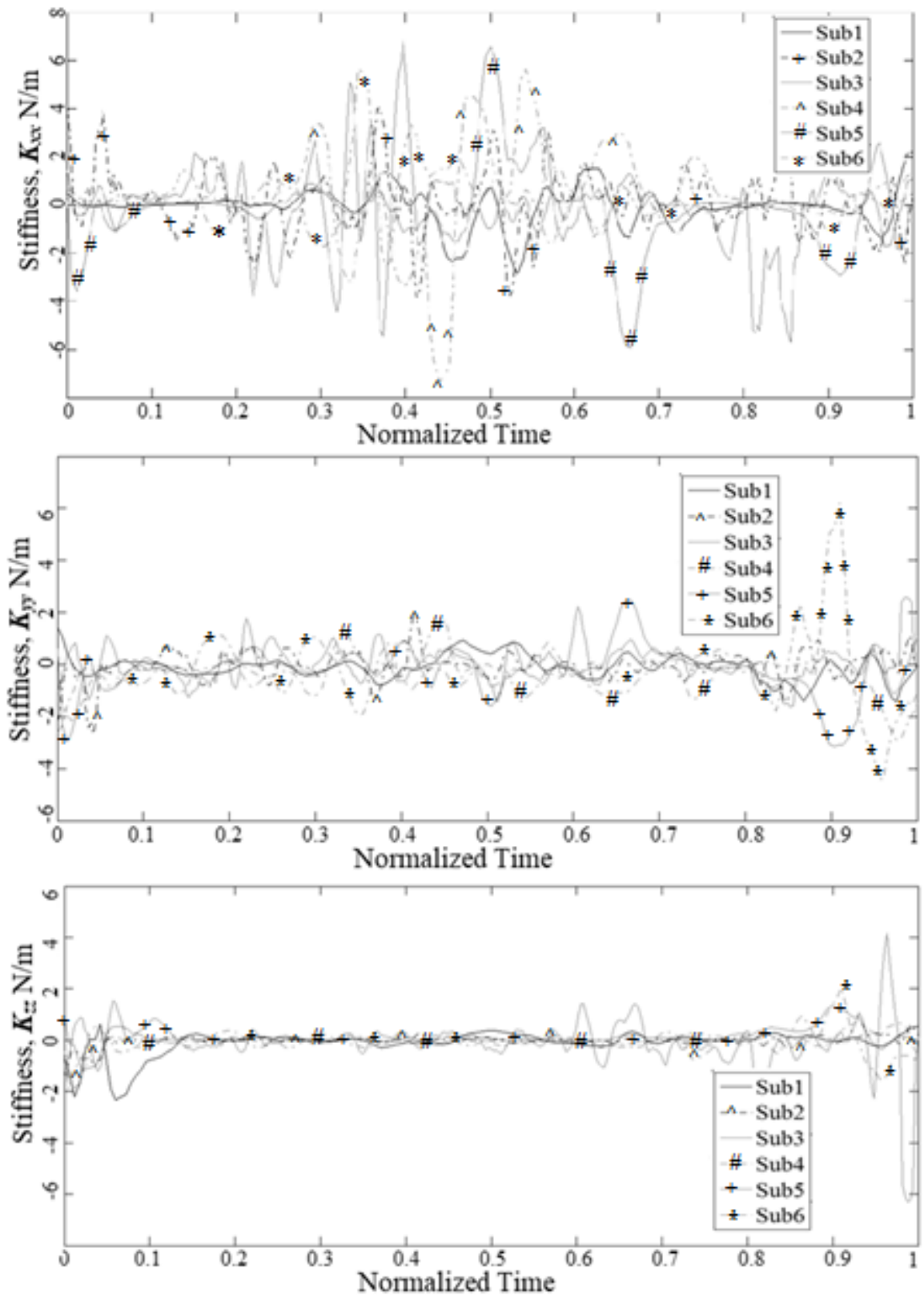

Figure 11. Stiffness components of all subjects

\subsection{Damping coefficient componenets}

The damping coefficient components of all dimension are shown in Figure 12. The magnitude of $Y$ diagonal component of damping factor is much smaller than the $X$ diagonal component of the damping factor. The maximum and minimum points are observed by subject 5 . For the $\mathrm{Z}$ diagonal damping factor observed during the starting and end of the motion, the average values lie between $100 \mathrm{~N} . \mathrm{s} / \mathrm{m}$ to $350 \mathrm{~N} . \mathrm{s} / \mathrm{m}$ for all subjects except subject 3 with the highest value of $500 \mathrm{~N} . \mathrm{s} / \mathrm{m}$. For the minimum, it is not the same average value for all subjects except subject 3 with around $-70 \mathrm{~N} . \mathrm{s} / \mathrm{m}$ to $-350 \mathrm{~N} . \mathrm{s} / \mathrm{m}$ and highest value at $-270 \mathrm{~N} . \mathrm{s} / \mathrm{m}$. The average and standard deviation is around $20 \mathrm{~N} . \mathrm{s} / \mathrm{m}$ to $40 \mathrm{~N} . \mathrm{s} / \mathrm{m}$ though with the peak for subject 4 and subject 3 at 67.70 N.s/m and 71.17 N.s/m, respectively. 
In this chapter the variation of impedance parameters is shown for different subjects with respect to time. From all the subjects it is seen that damping factor and stiffness are the mostly effected parameter during 3D hand movement. In all the studies it was observed that the magnitude changes of damping factor and stiffness are always high as compared to inertia. The magnitudes obtained for the impedances have shown similar trends as seen in other studies related to this field [24], [25].
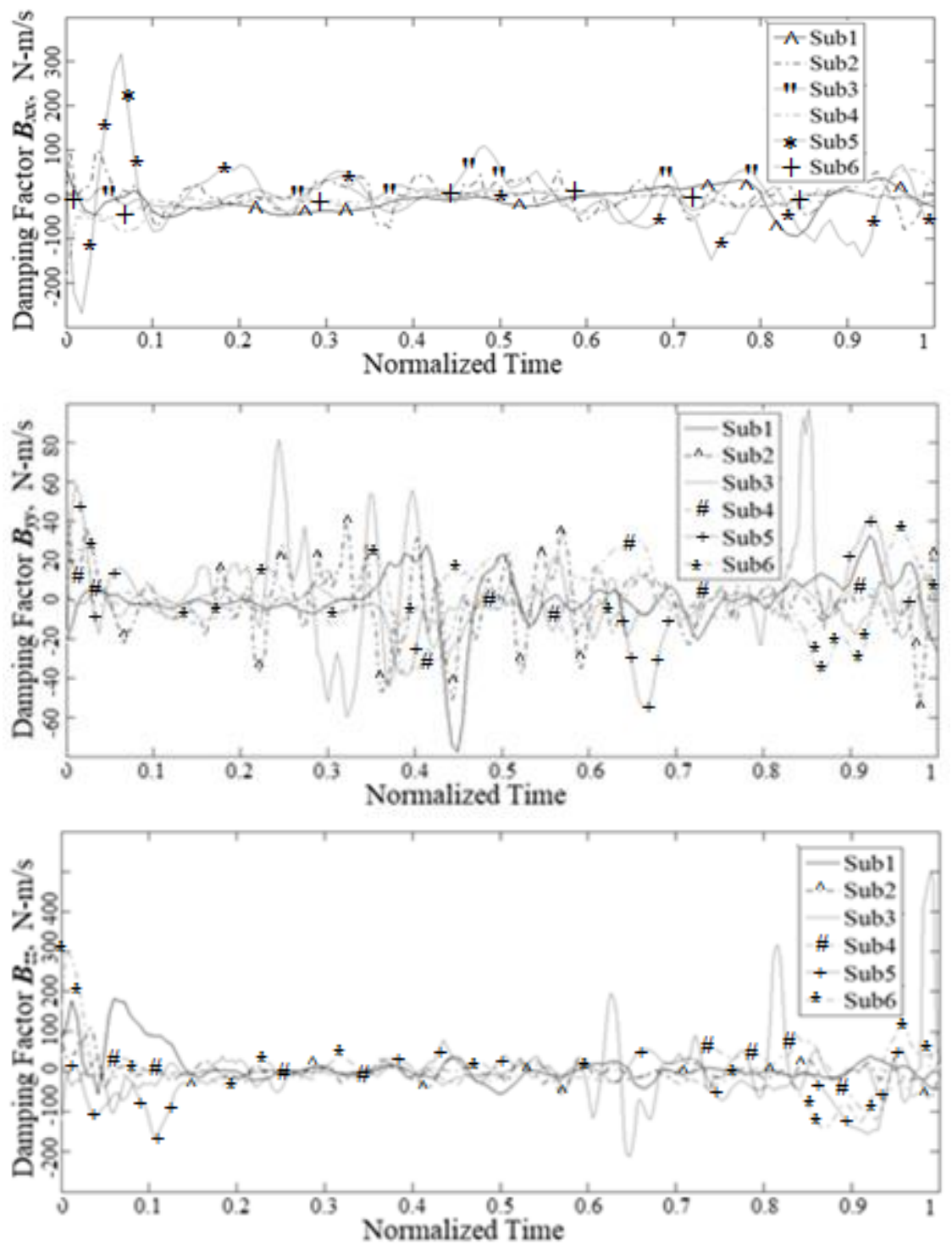

Figure 12. Damping factor of all subjects

\section{CONCLUSION}

The main purpose of this paper is to map the human arm impedance characteristics during daily active movements. In this research, 3D human arm movement was considered. Human arm was considered as mass-spring-damper system. The model for mass-spring-damper system was used to map impedance characteristics. The position, velocity, acceleration, and force were used to solve the model for impedance parameters e.g. inertia, damping factor, and stiffness. These impedance parameters were plotted against time. In this paper, positional data were measured by Measuring's Shape Tape device. During the experimentation, six subjects of age of 25 to 35 participated. Among them, four were female and two were male. Having analysed the impedance characteristics mapping, a mass-spring-damper system is found as appropriate model for mapping the human arm impedance characteristics. Mapping of dynamic impedance characteristics are done by plotting these characteristics against time. It is observed that the amounts of changes in damping 
factor and stiffness are considerable whereas very little change is observed in inertia. Mainly, these changes occur due to the arm movement.

\section{ACKNOWLEDGEMENTS}

Communication of this research is made possible through monetary assistance by Universiti Tun Hussein Onn Malaysia and the UTHM Publisher’s Office via Publication Fund E15216.

\section{REFERENCES}

[1] D. Rancourt and N. Hogan, "Dynamics of Pushing," Journal of Motor Behavior, vol. 33, no. 4, pp. 351-362, Dec. 2001, doi: $10.1080 / 00222890109601919$

[2] A. B. Slifkin and K. M. Newell, "Noise, information transmission, and force variability.," Journal of Experimental Psychology: Human Perception and Performance, vol. 25, no. 3, pp. 837-851, 1999, doi: 10.1037/0096-1523.25.3.837.

[3] A. Bicchi, S. L. Rizzini, and G. Tonietti, "Compliant design for intrinsic safety: general issues and preliminary design," in Proceedings 2001 IEEE/RSJ International Conference on Intelligent Robots and Systems. Expanding the Societal Role of Robotics in the the Next Millennium (Cat. No.01CH37180), vol. 4, pp. 1864-1869, doi: 10.1109/IROS.2001.976345.

[4] N. E. Budiyanta, L. Wijayanti, W. W. Basuki, H. Tanudjaja, and V. B. Kartadinata, "The development of healthcare mobile robot for helping medical personnel in dealing with COVID-19 patients," Indonesian Journal of Electrical Engineering and Computer Science, vol. 22, no. 3, p. 1379, Jun. 2021, doi: 10.11591/ijeecs.v22.i3.pp1379-1388.

[5] J. E. Colgate and N. Hogan, "Robust control of dynamically interacting systems," International Journal of Control, vol. 48, no. 1, pp. 65-88, Jul. 1988, doi: 10.1080/00207178808906161.

[6] F. Mussa-Ivaldi, N. Hogan, and E. Bizzi, "Neural, mechanical, and geometric factors subserving arm posture in humans," The Journal of Neuroscience, vol. 5, no. 10, pp. 2732-2743, Oct. 1985, doi: 10.1523/JNEUROSCI.05-10-02732.1985.

[7] C. H. Bennett and S. J. Wiesner, "Communication via one- and two-particle operators on Einstein-Podolsky-Rosen states," Physical Review Letters, vol. 69, no. 20, pp. 2881-2884, Nov. 1992, doi: 10.1103/PhysRevLett.69.2881.

[8] C. H. Bennett, G. Brassard, C. Crépeau, R. Jozsa, A. Peres, and W. K. Wootters, "Teleporting an unknown quantum state via dual classical and Einstein-Podolsky-Rosen channels," Physical Review Letters, vol. 70, no. 13, pp. 1895-1899, Mar. 1993, doi: 10.1103/PhysRevLett.70.1895.

[9] N. J. Miller, C. Rice-Evans, M. J. Davies, V. Gopinathan, and A. Milner, "A Novel Method for Measuring Antioxidant Capacity and its Application to Monitoring the Antioxidant Status in Premature Neonates," Clinical Science, vol. 84, no. 4, pp. 407-412, Apr. 1993, doi: 10.1042/cs0840407.

[10] H. Gomi and M. Kawato, "Human arm stiffness and equilibrium-point trajectory during multi-joint movement," Biological Cybernetics, vol. 76, no. 3, pp. 163-171, Apr. 1997, doi: 10.1007/s004220050329.

[11] E. Burdet, R. Osu, D. W. Franklin, T. Yoshioka, T. E. Milner, and M. Kawato, "A method for measuring endpoint stiffness during multi-joint arm movements," Journal of Biomechanics, vol. 33, no. 12, pp. 1705-1709, Dec. 2000, doi: 10.1016/S00219290(00)00142-1.

[12] E. Burdet, R. Osu, D. W. Franklin, T. E. Milner, and M. Kawato, "The central nervous system stabilizes unstable dynamics by learning optimal impedance," Nature, vol. 414, no. 6862, pp. 446-449, Nov. 2001, doi: 10.1038/35106566.

[13] H. Gomi and R. Osu, "Task-Dependent Viscoelasticity of Human Multijoint Arm and Its Spatial Characteristics for Interaction with Environments," The Journal of Neuroscience, vol. 18, no. 21, pp. 8965-8978, Nov. 1998, doi: 10.1523/JNEUROSCI.18-2108965.1998.

[14] M. A. Conditt, F. Gandolfo, and F. A. Mussa-Ivaldi, "The Motor System Does Not Learn the Dynamics of the Arm by Rote Memorization of Past Experience," Journal of Neurophysiology, vol. 78, no. 1, pp. 554-560, Jul. 1997, doi: 10.1152/jn.1997.78.1.554.

[15] R. Shadmehr and H. H. Holcomb, "Neural Correlates of Motor Memory Consolidation," Science, vol. 277, no. 5327, pp. 821825, Aug. 1997, doi: 10.1126/science.277.5327.821.

[16] S. N. Z. Ahmmad et al., "Objective assessment of surgeon's psychomotor skill using virtual reality module," Indonesian Journal of Electrical Engineering and Computer Science, vol. 14, no. 3, p. 1533, Jun. 2019, doi: 10.11591/ijeecs.v14.i3.pp1533-1543.

[17] D. W. Franklin, R. Osu, E. Burdet, M. Kawato, and T. E. Milner, "Adaptation to Stable and Unstable Dynamics Achieved By Combined Impedance Control and Inverse Dynamics Model," Journal of Neurophysiology, vol. 90, no. 5, pp. 3270-3282, Nov. 2003, doi: 10.1152/jn.01112.2002.

[18] K. P. Tee, E. Burdet, C. M. Chew, and T. E. Milner, "A model of force and impedance in human arm movements," Biological Cybernetics, vol. 90, no. 5, May 2004, doi: 10.1007/s00422-004-0484-4.

[19] S. K. Debnath, R. Omar, and N. B. A. Latip, "Comparison of different configuration space representations for path planning under combinatorial method," Indonesian Journal of Electrical Engineering and Computer Science, vol. 14, no. 1, p. 1, Apr. 2019, doi: 10.11591/ijeecs.v14.i1.pp1-8.

[20] M. Z. Al-Faiz and Y. I. Al-Mashhadany, "Analytical solution for anthropomorphic limbs model, (IK of human arm)," in 2009 IEEE Symposium on Industrial Electronics \& Applications, Oct. 2009, pp. 684-689, doi: 10.1109/ISIEA.2009.5356374.

[21] T. Asfour and R. Dillmann, "Human-like motion of a humanoid robot arm based on a closed-form solution of the inverse kinematics problem," in Proceedings 2003 IEEE/RSJ International Conference on Intelligent Robots and Systems (IROS 2003) (Cat. No.03CH37453), vol. 2, pp. 1407-1412, doi: 10.1109/IROS.2003.1248841.

[22] Saeed B. Niku, Introduction to Robotics: Analysis, Control, Applications, 3rd Editio. John Wiley \& Sons, 2020.

[23] M. Katayama and M. Kawato, "Virtual trajectory and stiffness ellipse during multijoint arm movement predicted by neural inverse models," Biological Cybernetics, vol. 69, no. 5-6, pp. 353-362, Sep. 1993, doi: 10.1007/BF01185407.

[24] M. M. Rahman, M. H. Rahman and R. Ikeura, "Impedance Characteristic of Shoulder and Elbow Joints during Passive Movement," First International Conference on Industrial and Information Systems, 2006, pp. 231-235, doi: 10.1109/ICIIS.2006.365729.

[25] P. K. Artemiadis, P. T. Katsiaris, M. V. Liarokapis and K. J. Kyriakopoulos, "Human arm impedance: Characterization and modeling in 3D space," 2010 IEEE/RSJ International Conference on Intelligent Robots and Systems, 2010, pp. 3103-3108, doi: $10.1109 /$ IROS.2010.5652025. 


\section{BIOGRAPHIES OF AUTHORS}
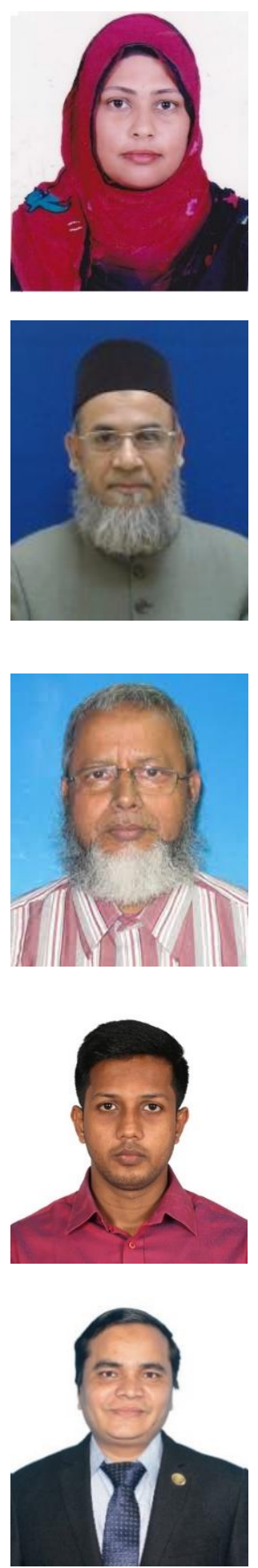

Tasnuva Tabashhum Choudhury (iD) $\mathrm{SC}$ P received his B.Sc. degree in Mechanical Engineering from Rajshahi University of Engineering and Technology (RUET) in 2005. After this she completed her MSc degree from International Islamic University (IIUM), Malaysia in Mechatronics Engineering in 2015. She has been an assistant professor of Mechanical Engineering with Rajshahi University of Engineering and Technology (RUET), since 2007. She is currently persuing her PhD in Saitama University, Japan. Her research interest includes robotics, human-robot cooperation, motor learning, engineering mechanics, and solid mechanics. She can be contacted at email: ttc.ruet@ gmail.com.

Md Mozasser Rahman (ID 8d SC P currently is an Associate Professor in the Department of Mechanical Engineering Technology, Universiti Tun Hussein Onn Malaysia (UTHM). Dr. Mozasser received a B. Sc. Eng. degree from Bangladesh Institute of Technology (BIT) Khulna in Mechanical Engineering in 1988. After graduation he worked for the same institute as a lecturer. He got practical knowledge and experiences in industrial maintenance and automation. He was later conferred a M. Eng. degree and Ph. D. from Mie University, Japan, in 2000 and 2003 respectively. Dr. Mozasser has expertise in Robotics and Industrial Automation. His research area covers human-robot cooperation, movement characteristics of human arm, artificial and human organ. He is a Member of the Institution of Mechanical Engineers, UK and a Chartered Engineer registered with the Engineering Council, UK. He can be contacted at email: mozasser@uthm.edu.my.

Md Raisuddin Khan (D) SC P received his B.Sc. degree in Mechanical Engineering from the former Engineering College, Rajshahi, Bangladesh [(currently known as Rajshahi University of Engineering and Technology (RUET)], in 1983. He received his M. Sc and Ph. $\mathrm{D}$ degrees in Mechanical Engineering from the Bangladesh University of Engineering and Technology (BUET) in 1988 and 1996 respectively. Currently he is a Professor in the department of Mechatronics Engineering of the International Islamic University Malaysia (IIUM). He has been serving the Mechatronics Engineering Department of IIUM for more than sixteen years. He has so far published more than sixty papers in different international journals and conferences. He is life fellow of IEB and BSME, and senior member of IEEE. He can be contacted at email: raisuddin@iium.edu.my.

Fazlur Rashid (D) 8f SC P received a BSc degree in Mechanical Engineering from Rajshahi University of Engineering \& Technology (RUET), Bangladesh, in 2016. He is a faculty member (Lecturer) in the Department of Mechanical Engineering at RUET. He is currently persuing his $\mathrm{PhD}$ in Missouri University of Science and Technology. His research interests include energy, control system, energy harvesting systems, and biofuels. He worked on a research project in the control engineering lab at the Department of Mechanical Engineering, Saitama University, Japan in 2019. He can be contacted at email: frrashid10@ gmail.com.

Md. Rabiul Islam Sarker (iD S SC P is actively engaged in a teaching position in the Faculty of Mechanical Engineering since 2006, following completion of his undergraduate degree. He completed his M. Sc Engineering degree from RUET and PhD degree from the University of Adelaide Australia. In his professional career, he has been joined in the positions of Lecturer, Assistant Professor, Associate Professor and Professor respectively. He can be contacted at email: islamrabiul@me.ruet.ac.bd 